This is the final peer-reviewed accepted manuscript of:

Ballestra LV, Guizzardi A, Palladini F. Forecasting and trading on the VIX futures market: A neural network approach based on open to close returns and coincident indicators. Int J Forecast. 2019;35(4):1250-1262.

The final published version is available online at:

https://doi.org/10.1016/j.ijforecast.2019.03.022

Rights / License:

The terms and conditions for the reuse of this version of the manuscript are specified in the publishing policy. For all terms of use and more information see the publisher's website.

This item was downloaded from IRIS Università di Bologna (https://cris.unibo.it/)

When citing, please refer to the published version. 


\title{
Forecasting and trading on the VIX futures market: a neural network approach based on open to close returns and coincident indicators
}

\author{
Ballestra Luca Vincenzo ${ }^{1}$, Andrea Guizzardi ${ }^{1}$, Fabio Palladini ${ }^{1}$
}

${ }^{1}$ Department of Statistical Science, University of Bologna.

\begin{abstract}
Previous work has highlighted the difficulty of obtaining accurate and economically significant predictions of VIX futures prices. We show that both low prediction errors and a significant amount of profitability can be obtained by employing a neural network model to predict VIX futures returns. In particular, we focus on open to close returns (OTCRs) and we consider intraday trading strategies, taking into account non-lagged exogenous variables that closely reflect the information possessed by traders at the time they decide to invest. The neural network model with only the most recent exogenous variables (namely, the return on the Indian BSESN index) is superior to an unconstrained specification with ten lagged and coincident regressors, which is, actually, a form of weak efficiency involving markets of different countries. Moreover, the neural network reveals to be more profitable than both a logistic specification and heterogeneous autoregressive models.
\end{abstract}

Keywords: VIX, VIX futures, forecasting, coincident indicators, trading strategies, weak efficiency

\section{Introduction}

The VIX index represents the market's estimate of the future volatility of the S\&P 500 over the next thirty days. It provide a benchmark of the short-term expected volatility, as futures and options contracts can be inscribed on (see Whaley, 2008). In fact, implied volatility reflects the market makers' point of view about the expected volatility of the futures' underlying assets. Therefore, since market makers are often among the most informed agents, implied volatility should outperform the historical one in forecasting the realized volatility of the futures' underlying asset (Shu and Zhang, 2012).

Despite the importance and the common use of VIX as a volatility measure, only little attention has been paid to the problem of forecasting it. In particular, the few works on the subject show that the VIX is to some extent predictable. This finding, albeit theoretically interesting, is not necessarily helpful for traders, because VIX is tradable only as derivative contracts, whose dynamics does not always follow that of the VIX index. For example, Asensio (2013), Degiannakis (2008), Kostantinidi et al. (2008), Konstantinidi and Skiadopoulos (2011), who are among the few authors focusing on VIX futures (henceforth referred to as VXFs), highlight only a weakevidence of statistical predictability and experience a low level of profitability whenimplementing trading strategies based on VIX forecasts. The overall picture is not encouraging for investors: on the one hand, there is evidence that VIX is predictable; on the other, it seems very hard to trade VXFs by learning from the (predicted) VIX dynamics.

In the present paper, in order to fill this "forecasting gap", we present a new approach for modelling VXF returns that provides a significant amount of predictability and allows us to build profitable trading strategies.

Specifically, we rely on a feed-forward neural network model, which yields a very general form of nonlinearity. Moreover, we consider exogenous variables that closely reflect the information possessed by traders at the time they decide to invest. In particular, in the information set we include non-lagged exogenous variables that are available only a few hours before the Chicago Board Options Exchange (CBOE) opening. 
Instead of forecasting VXF daily returns (DRs), we predict VXF open to close returns (OTCRs), which are free of spurious effects related to trading timing (Anderson et al., 2012). No less importantly, by considering intraday returns we can easily connect forecast performances to the profits earned by those investors who open and close a position on the same day, taking advantage of the fact that stock volatility is substantially higher intraday than overnight (Muravyev and Ni, 2016).

The contribution of the present paper is fourfold: first, we show that, by using an appropriate modelling approach, accurate predictions of OTCRs on VFX can be obtained.

Second, we show that a neural network model whose only input variable is the most recent exogenous one (namely, the return on the BSESN index) is superior to an unconstrained model with ten lagged and coincident regressors. That is, VXF prices strongly reflect the most recent publicly available information, which is, actually, a form of weak efficiency involving markets of different countries.

Third, we compare the VXF OTCR prediction provided by the neural network model with that yield by a logistic specification, by a simple (Naïve) model always forecasting negative VXF OTCRs, and by both a heterogeneous autoregressive (HAR) and two augmented heterogeneous autoregressive (HAR_X) models. The results obtained reveal that the neural network significantly outperforms all the other models as far as mean directional accuracy is concerned.

Fourth, we simulate and test various trading strategies, with different abilities to filter out false signals. Again, the predictions of the neural network model turn out to be more profitable than those of the rival models.

The remainder of the paper is organized as follows. Section 2 describes the main issues related to predicability and profitability in the VIX/VXF market. Section 3 presents the model specifications, as well as the measures of forecast accuracy and profitability. Section 4 shows and discusses the main estimation results, focussing on the comparative assessment of the models' predictions and on the profitability of the corresponding trading strategies (considering both VXF OTCRs and VFX DRs). Finally, Section 5 concludes.

\section{Main issues related to predictability and profitability in the VIX/VXF market}

Research on VIX has been largely dominated by autoregressive conditional heteroscedasticity models taking into account non-linearity, long memory features and/or lagged exogenous variables. Ahoniemi (2006) tests and compares the predictive capabilities of probit, ARIMAX-GARCH and ARFIMA models. By considering a large set of U.S. financial and macroeconomic variables, she finds that the addition of exogenous regressors enhances forecasting performance, whereas improvements from adding GARCH errors or long memory features are negligible. Degiannakis (2008) introduces a threshold effect to model asymmetry, but no incremental information in forecasting VIX is obtained. Konstantinidi et al. (2008) model several implied volatility indices, including VIX, in a multivariate VAR framework, which, however, does not yield any significant improvement in forecasting. Long memory features are also exploited, among others, by Fernandes et $a$ l. (2014), who apply a heterogeneous autoregressive (HAR) model coupled with a neural network to better capture non-linearities. Nevertheless, they find only little evidence of non-linearity, since the HAR model augmented by the neural network performs as well as the linear HAR model with no neural network. Psaradellis and Sermpinis (2016) analyze three volatility indices including VIX, and, by employing support vector regression models coupled with a genetic algorithm, find significant evidence of strong non-linearities .

Other approaches look at both VIX and VXF prices, with the aim to study causality direction, and/or to investigate the forecast accuracy and the profitability of trading the VXFs. Shu and Zhang (2012) find that VXF prices drive spot VIX if a linear model is employed. However, after searching for non-linear relationships, both spot and futures prices react simultaneously to the arrival of new information. Jablecki et al. (2015) and Luo and Zhang (2012) show that the shape of the implied volatility term structure and the volatility risk premium help in forecasting VIX. 
A very important point to remark is that the predictability of the VIX index does not necessarily imply that VXF prices can be predicted too. This is clearly pointed out by Degiannakis (2008), who observes that in the $26 \%$ of the trading days the log-returns of VIX and of its futures have opposite signs. He concludes that "an agent cannot utilize VIX predictions in creating abnormal returns in implied volatility futures markets", and highlights the need for future work focusing directly on the predictability of VXFs. As well, Degiannakis (2008), Kostantinidi et al. (2008), Konstantinidi and Skiadopoulos (2011), despite finding some evidence of predictability of the VIX index, do not succeed in using VIX forecasts toconstruct profitable trading strategies.

Asensio (2013) addresses the topic from a more theoretical point of view, and, to stress out the complexity of the VIX/VXF market, talks about a "VIX-VFX Puzzle". In particular, he identifies a number of factors that cause VXFs to be "consistently overpriced relative to the subsequent moves in the underlying VIX index".

To provide an intuition of the VIX-VXF Puzzle, we have computed the correlation between the DRs on VIX and the DRs on the VXF on the time interval from March 26, 2007 to December 20, 2016. For the VXF, we use the continuous time series provided Thomson Reuters Datastream (Type 0), which contains the prices of either nearest contract month futures or second nearest contract month futures (more details about time series construction can be found in Thomson Reuters Datastream(2010)). We found that the correlation is 0.823 when considering the whole data set (2454 observations), and falls down to 0.783 when taking the average of the correlations computed on monthly sub-samples (in the chosen time interval we have 116 monthly subsamples). Furthermore, in Figure 1.a we show how the monthly correlations varies over time. As we may observe, the profile is quite erratic, with many spikes around 0.4 and a minimum close to 0.2 .

If we consider the correlation between VIX OTCRs and VXF OTCRs, the trading gap between VIX and VXF is still more serious: the correlation is 0.767 on the whole data set, and falls to 0.771 if we compute the average of the correlations on the 116 monthly sub-samples. Moreover, as shown in Figure 1.b, the correlation dynamics is still very erratic.

Therefore, since the dynamics of VXFs does not closely reflect that of the VIX, even very accurate predictions of the VIX may not allow an investor to earn significant profits by trading VIX futures as highlighted in the literature.

We shall acknowledge that, according to Psaradellis and Sermpinis (2016), VIX forecasts can yield a "noteworthy prospect" of achieving economically significant profits in the VXFs market. In particular, they apply a non-linear long-memory model to predict the VIX, and then they go long (short) in the VXF when the forecasted value of the index is greater (smaller) than its current value. Nevertheless, even if Psaradellis and Sermpinis succeeded in constructing profitable trading strategies, due to the poor and erratic correlation between the VIX and its futures, an investor who trades VXFs based on VIX forecasts could fail to obtain significant profits anyway.

Figure 1. Corre lation be tween VIX and VXF (computed on monthly sub-samples). DRs returns (a); OTCRs (b)

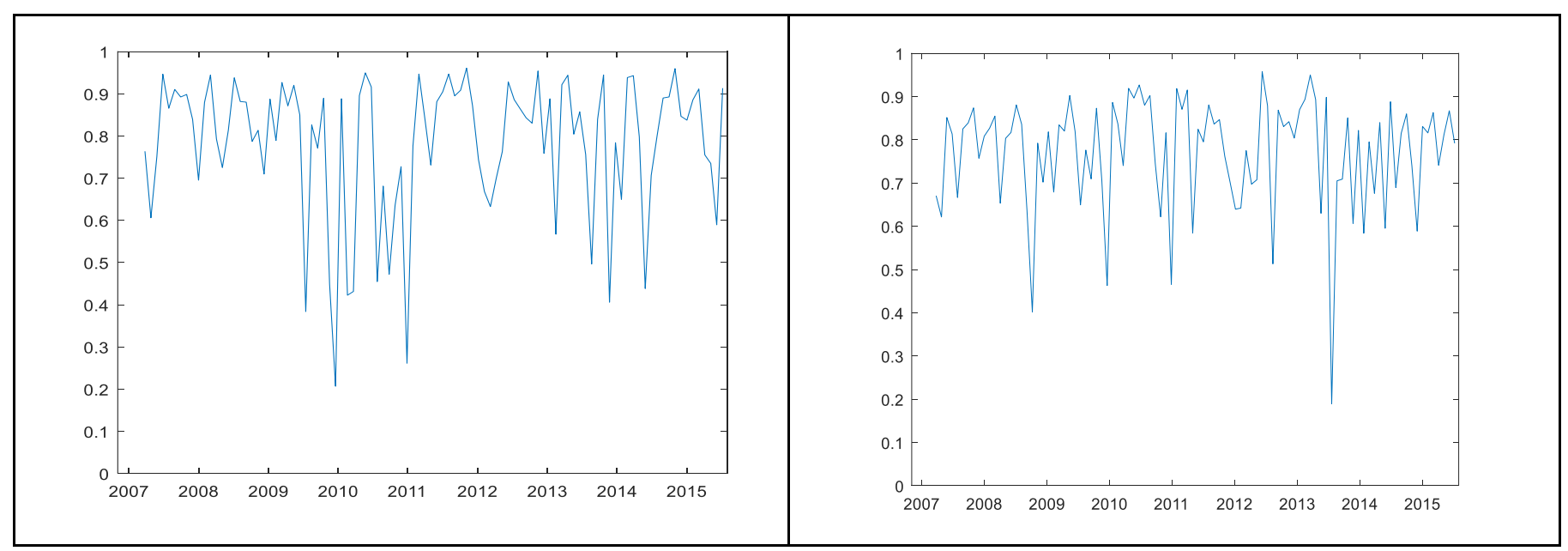


To fill the trading gap between VIX and VXF, Jablecki at al. (2014) propose an original and interesting approach that takes into account both the current level of VIX and the volatility term structure. However, even if they succeed in building some profitable trading strategy, their predictions of VXF levels do not outperform naïve forecasts.

To overcome the non-tradability of the VIX index, as well as to avoid the use of VIX futures, some authors (see Ahoniemi, 2006 and Degiannakis et al., 2018) propose trading sessions based on buying/selling straddles of options on the S\&P 500. The predictions on the VIX drive the decision to buy or sell the straddle, and, in particular, a long/short position is taken if the VIX is expected to rise/fall. However, such an approach is guaranteed to be profitable only if the strikes of the traded straddles coincide with the S\&P (i.e. the straddles are delta-neutral), which cannot always be the case due to the limited number of straddle strikes that are available on the market.

Finally, while some authors have already focused on the predictability of DRs on VXFs, no one has ever tested either the predictability of opening to close returns on VXFs or the profitability of the related trading strategy that amounts to opening and closing a position on the same day.

\section{Methodology and data}

In order to bypass the complex, and, at least to some extent, non-predictable relationship between the VIX and its futures, we directly model the VXF dynamics. We use a neural network approach, which, as suggested by the literature, appears to be more successful than a (linear) time series approach in anticipating the evolution of the implied volatility.

Furthermore, the choice of the exogenous variables is very important too. When modelling financial phenomena, it is common practice to take the information set from the same market to which the variables being explained belong. Nevertheless, as suggested by Shen et al. (2012), under weak efficiency hypothesis, price dynamics in markets that close right before or at the very beginning of U.S. trading, should incorporate much more information than lagged variables on the U.S. market. Therefore, we augment the information set by some "coincident indicators" taken from Asian stock exchange markets leveraging the time zone difference.

The VIX index is calculated using options with two consecutive expirations having more than 23 days and less than 37 days to expiration (for further information see CBOE, 2015). Typically, once a week, some of the options used for the calculation start having less than 24 days to expiration, and thus they are rolled to new maturities. When this happens the VIX index usually experiences a jump, and, consequently, if returns are computed as the log closing price difference between two consecutive days, some bias arises.

To avoid this problem, we set our dependent variable as the VXF open-to-close return (OTCR), which is calculated as $\ln \left(\right.$ close $\left._{t}\right)-\ln \left(\right.$ open $\left._{t}\right)$. OTCRs offer the advantage of taking into account only the "genuine" autocorrelation that arises from partial price adjustment and time-varying risk premia (Anderson et al., 2012), and incorporate the relevant information about the variability of financial assets, since stock volatility is substantially higher intraday than overnight (Muravyev and Ni, 2016). No less importantly for the purposes of the present work, the use of intraday returns allows to easily connect the forecasting performances to the profits earned by a trader who opens and closes a position on the same day. In this respect, it is also worth observing that, from the practical standpoint, a trading strategy that consists on buying/selling a VXF is not affected by small liquidity issues, since, the liquidity of futures' market has considerably grown over the years (Shu and Zhang, 2012).

With respect to the usual approach based on the log difference between subsequent closing prices, we acknowledge that we do not measure any overnight gap. Nevertheless, this does not represent a limit, because we consider a trading strategy that open and close the positions within the same day. 


\subsection{Models specification}

Both the VFX and the VIX, as well as the variables used to explain them (see Section 3.3), are collected at a set of (consecutive) trading days $1,2, \ldots, T_{3}$ (so that $T_{3}$ denotes the size of our dataset). Then, we fix two positive integers $T_{1}$ and $T_{2}$, such that $T_{1}<T_{2}<T_{3}$, in order to form three sub-samples. Precisely, the training set, containing the data observed at days $1,2, \ldots, T_{1}$, is used to estimate the econometric models. The validation set, containing the data observed at days $T_{1}+1, T_{1}+2, \ldots, T_{2}$, is used to train the neural network, so as to minimize overfitting problems (that is, to reach a trade-off between model complexity and expected forecasting accuracy). The first $T_{2}$ observations are also used for optimizing the parameters of the employed trading strategy.

A third subset, the test set, containing the data observed in $T_{2}+1, T_{2}+2, \ldots, T_{3}$, is exploited to assess both the ex-post forecast performance of the models and the profitability of the resulting trading strategies. We model intraday returns on the VXF by means of a multilayer augmented feed-forward neural network, a black-box approach proved to be able to approximate complex (non-linear) relationships (see Thenmozhi, 2006).

We specify a single hidden layer neural network. The input layer is made by $S$ nodes, or neurons, that correspond to the explanatory variables. A constant term $w_{, 0}$ (the so-called bias) is also included. These input terms are first multiplied by a matrix $W$ of weights and then transformed by a non-linear function (the so-called transfer or activation function, which we denote by $f$ ):

$$
h_{j}\left(x_{1}, x_{2}, \ldots, x_{S}\right)=f\left(w_{j, 0}+\sum_{i=1}^{S} w_{j, i} x_{i}\right), \quad j=1,2, \ldots, J,
$$

where $J$ is the number of neurons in the hidden layer, to be selected according to the parsimony principle (so as to achieve the best trade-off between complexity and forecasting accuracy). Following a common approach, the activation function is chosen to be the hyperbolic tangent function:

$$
f(z)=\frac{e^{z}-e^{-z}}{e^{z}+e^{-z}}
$$

Then, as happens for input nodes, the hidden neurons' output is sent to the output layer, multiplied by a second matrix $V$ of weights and transformed by a non-linear function:

$$
\operatorname{out}_{n}\left(h_{1}(\cdot), h_{2}(\cdot), \ldots, h_{j}(\cdot)\right)=f\left(v_{n, 0}+\sum_{j=1}^{J} v_{n, j} h_{j}(\cdot)\right), \quad n=1,2,
$$

where out is a two dimensional vector representing the final neural network prediction, in particular if $o u t_{1}<$ $o u t_{2}$ the OTCR VFX is forcasted down, whereas if $o u t_{1}>o u t_{2}$ the OTCR VFX is forcasted up. Normally, each node in a given layer is connected to all the nodes. Given the number of hidden layers (which in our case is one), the complexity of the model and its capability to approximate the input depend on the number of neurons $J$. However, parsimony is usually seen as the leading principle for model specification as complexity increases both the risk of overfitting and the computational time. Once the structure of the neural network is created, the parameters are estimated by minimizing a suitable loss function, which is done by applying a numerical optimization algorithm (in the network terminology, we say that the network is trained with a learning algorithm). The loss function we choose, which is very common for our two-class problem, is the "softmax cross-entropy":

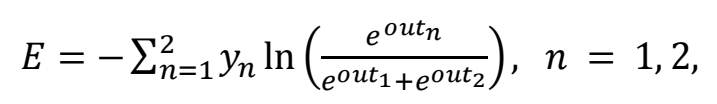

and either $\left(y_{1}, y_{2}\right)=(0,1)$ if the observed direction of the OTCR VFX is down or $\left(y_{1}, y_{2}\right)=(1,0)$ if the observed direction of the OTCR VFX is up. The problem of bad local minima is dealt with by considering different starting points in the learning phase.

To evaluate whether the complexity and non-linearity implied by the neural network approach worth it, the following logistic regression is used as a benchmark to compare with: 


$$
\pi(x)=\frac{e^{\beta_{0}+\sum_{j=1}^{S} \beta_{j} x_{j}}}{1+e^{\beta_{0}+\sum_{j=1}^{S} \beta_{j} x_{j}^{\prime}}}
$$

where $\pi(x)$ denotes the probability that the future observation is positive and $x_{1}, x_{2}, \ldots, x_{s}$ are the same lagged endogenous and/or exogenous variables that we employ in the neural network model.

Moreover, always for comparison purposes, we consider a simple model (Naïve) that always predicts VXF OTCRs to be negative (since the empirical examination of the VXF time series shows that negative OTCRs are more likely to occur than positive OTCRs).

Finally, to compare with a more conventional approach, we also try to forecast VXF movements based on VIX predictions. Specifically, we use a Heterogeneous Autoregressive model of Realized Volatility (HAR) and an augmented HAR (HAR_X) to predict VIX. The HAR model, which is originally due to Corsi (2009), and the HAR_X model are often employed for predicting the volatility of financial time series, since they are parismonious approaches and can capture long-memory effect (see, e.g., Busch et al. 2011, Degiannakis and Filis, 2017, Degiannakis et al., 2018, Fernandes et al., 2014).

According to the HAR model, the realized volatility $(R V)$ is predicted as follows:

$$
R V_{t}=b_{0}+b_{1} R V_{t-1}+b_{2} M A(R V)_{t-1}^{5}+b_{3} M A(R V)_{t-1}^{22}+\epsilon_{t}
$$

where

$$
M A(R V)_{t-1}^{5}=\frac{1}{5} \sum_{k=1}^{5} R V_{t-k}, \quad M A(R V)_{t-1}^{22}=\frac{1}{22} \sum_{k=1}^{22} R V_{t-k}
$$

Instead, the HAR_X specification predicts the realized volatility by taking also into account the current value of $S$ exogenous regressors:

$R V_{t}=b_{0}+b_{1} R V_{t-1}+b_{2} M A(R V)_{t-1}^{5}+b_{3} M A(R V)_{t-1}^{22}+\sum_{i=1}^{S}\left(c_{1, i} x_{i, t}+c_{2, i} M A\left(x_{i}\right)_{t}^{5}+c_{3, i} M A\left(x_{i}\right)_{t}^{22}\right)$,

where $M A\left(x_{i}\right)_{t}^{5}$ and $M A\left(x_{i}\right)_{t}^{22}$ are defined analogously to (7). The index $t$ in the exogenous variables does not imply using future data that do not belong to the current information set. In fact, the exogenous regressors refer to markets closing before the VFX's opening (see Section 4.1) and thus they become available at least 4 hours in advance.

The HAR and HAR_X models are specifically designed for forecasting realized volatility and thus they cannot be directly applied to VXF returns. Therefore, in place of RV we consider VIX. By doing this, we predict the VIX based on either (6) or (8), and then we forecast the future direction of the VXF OTCR as follows:

$$
\left\{\begin{array}{l}
V X F_{t}-V X F_{t}<0 \text { if } V X F_{t}-V X F_{t}<0 \\
V X F_{t}-V X F_{t}>0 \text { if } V X F_{t}-V X F_{t}>0 \\
V X F_{t}-V X F_{t}=0 \text { if } V X F_{t}-V X F_{t}=0 .
\end{array}\right.
$$

\subsection{Forecast accuracy}

To measure forecast accuracy, one can use either a loss function based on the magnitude of the forecasting error, such as the mean square forecasting error (MSFE) and the mean absolute forecasting error (MAFE), or, 
instead, a classification loss function (directional forecasting). If the former approach is the most popular in the literature, the latter allows for a better assessement of potential profitability. In fact, as shown by Leitch and Tanner (1995), Diebold and Mariano (1995) and Granger and Pesaran (2000) among others, directional accuracy (DA) is more connected with profits than standard accuracy measures such as MSFE. In addition, Blaskowitz and Herwartz (2011) emphasize the robustness of DA in the presence of signal bias and outliers. Finally, some papers (see,e.g., Degiannakis, 2008 and Costantini et al., 2016) combine DA with the profit/loss of a trading strategy, and obtain decision-based loss functions that allow one to assess accuracy in economic terms.

Therefore, in the present paper we employ the mean directional accuracy, which, for the $k$-th model (we are going to compare six different models) is computed as follows:

$$
M D A_{k}=\frac{1}{T_{3}-T_{2}} \sum_{t=T_{2}+1}^{T_{3}} \mathbf{1}_{\operatorname{sign}\left(O T C R_{t}\right)=\operatorname{sign}\left(\widehat{O T C R}_{k, t}\right)},
$$

where $\widehat{\operatorname{TTCR}}_{k, t}$ denotes the OTCR at day $t$ forecasted by the $k$-th model, and $\mathbf{1}$ an indicator function that is equal to one if the two signs coincide, and zero otherwise.

We test directional forecasting accuracy with the market-timing test for predictive accuracy (Pesaran and Timmermann, 1992). The null hypothesis is that the predicted and the realized signs are independent, i.e. the forecasted market directions do not inform on the sign of the realized returns. Granger and Pesaran (2000) provide the following version of the test:

$$
P T=\frac{\sqrt{T_{3}-T_{2}}\left(\frac{N_{p p}}{N_{p p}+N_{n p}}-\frac{N_{p n}}{N_{p n}+N_{n n}}\right)}{\left(\frac{\hat{\pi}_{f}\left(1-\hat{\pi}_{f}\right)}{\hat{\pi}_{o}\left(1-\hat{\pi}_{o}\right)}\right)^{1 / 2}}
$$

where the subscripts $p$ and $n$ indicate positive and negative VXF returns, respectively, $N_{p n}$ is the number of times the VXF return was negative and the forecast was positive, and $N_{p p}, N_{n n}, N_{n p}$ are defined accordingly. Moreover, $\hat{\pi}_{o}=\frac{N_{p p}+N_{n p}}{T_{3}-T_{2}}$ is the probability that returns are positive and $\hat{\pi}_{f}=\frac{N_{p p}+N_{p n}}{T_{3}-T_{2}}$ is the probability that returns are forecasted to be positive. As shown by Granger and Pesaran (2000), under the null hypothesis that the predicted and the realized signs are independent, $P T$ has a standard normal distribution (with zero mean and unitary variance). Thus, we can easily test if the predicted and the realized signs are independent by comparing with the quantile of the standard normal distribution.

We devote a special attention to data-snooping biases, a common problem in inference with non-linear models because of the many degrees of freedom that are lost. Therefore, in order to assess if the predictive superiority of the neural network is systematic and not due to luck, we assess the predictive performance of this highly parametrized non-linear specification by applying a Monte Carlo cross validation technique. Specifically, we consider 1000 random permutations of the sequence of days $1,2, \ldots, T_{3}$ in which data are observed. In each permuted sequence we form the training set with the data at places $1,2, \ldots, T_{1}$, the validation set with the data at places $T_{1}+1, T_{1}+2, \ldots, T_{2}$, and the test set with the data at places $T_{2}+1, T_{2}+2, \ldots, T_{3}$. By doing that, for each of the 1000 permutations we have a different (random) distribution of all the economic variables among the training, validation and test sets. Then, we check the distribution of the mean directional accuracy of the simulated neural network model over the set of Monte Carlo permutations.

Furthermore, we also evaluate the superior predictive ability (SPA) of the rival models applying the test developed by Hansen (2005), which is briefly described in the following. We consider, in turn, each model as the benchmark to compute the following relative performance at time $t$ of model $k$ :

$$
d_{k, t} \equiv L\left(\operatorname{sign}\left(O T C R_{t}\right), \operatorname{sign}\left(\widehat{O T C R}_{0, t}\right)\right)-L\left(\operatorname{sign}\left(O T C R_{t}\right), \operatorname{sign}\left(\widehat{O T C} R_{k, t}\right)\right)
$$


where $k=0$ refers to the model chosen as the benchmark, whereas $k=1,2, \ldots$ refer to the rival models and $L(\cdot)$ is a given loss function. Let us consider the sample average of $d_{k, t}$ :

$$
\bar{d}_{k}=\frac{1}{T_{3}-T_{2}} \sum_{t=T_{2}+1}^{T_{3}} d_{k, t}
$$

and let us define:

$$
A_{k}=\sqrt{T_{3}-T_{2}} \cdot \bar{d}_{k}
$$

Moreover, let $\widehat{\omega}_{k}$ denote a consistent estimator of the standard deviation of $A_{k}$. Then, the null hypothesis of the test is that the predictive ability of the benchmark is superior to that of the other five models. Such an hypothesis is rejected based on the significance of the studentized test statistic

$$
T^{S P A} \equiv \max \left[\max _{k=1,2, \ldots} \frac{A_{k}}{\widehat{\omega}_{k}}, 0\right] .
$$

Suitable p-values for the statistics $T^{S P A}$ are calculated based on bootstrap resamples.

The trading strategy we consider is as follows: at day $t$, depending on the forecasted value of $O T C R_{t}$, either we do nothing or we take a long/short position on the VXF when the market opens and liquidate it when the market closes. Accordingly, we measure the total profit in the time interval from day $T_{2}+1$ to day $T_{3}$ by using the cumulative directional value:

$$
C D V=\sum_{t=T_{2}+1}^{T_{3}} D V_{t}
$$

where

$$
D V_{t}=O_{t} \cdot O T C R_{t}
$$

with $O_{t}=1,-1$, or 0 if at day $t$ we take a long, a short or a flat position, respectively. The Hansen test described above is also used to assess the superior ability of the models in generating profits. In particular, we use the opposite of the directional value (17) as a loss function in (12).

\subsection{Dataset}

We take into account the VIX futures continuous time series constructed by Thomson Reuters Datastream (Type 0), which contains the prices of either the nearest contract month futures or the second nearest contract month futures. We consider daily data from March 26, 2007 to September 30, 2017, removing days with no value (e.g. holidays). With this choice, we collect $T_{3}=2639$ observations on the whole dataset. To form the training, validation and test sets introduced in Section 3.2, we set $T_{1}=1718, T_{2}=2086$ (i.e., the training set contains data from March 26, 2007 to January 21, 2014, the validation set contains data from January 22, 2014 to July 8, 2015, the test set contains data from July 9, 2015 to September 30, 2017).

We compute the logarithmic OTCR series of both VIX and VXF. Descriptive statistics (for the sub-sample made of the first $T_{2}$ observations) are provided in Table 1.

As is typical of many financial time series, the distributions of log-returns are slightly asymmetric and show a kurtosis greater than three. The Jarque-Bera $(\mathrm{JB})$ normality test always allows to reject normality $(p<5 \%)$. The time series of both VIX and VXF returns are stationary as indicated by the Augmented Dickey-Fuller (ADF), Philips-Perron (PP) and KPSS tests. However the futures oscillates less than its underlying (i.e. the difference between maximum and minimum is smaller). 
Table 1: Descriptive statistics on OTCR

\begin{tabular}{c|c|c}
\hline \hline & VIX & VXF \\
\hline Mean & -0.0056 & -0.0010 \\
\hline Median & -0.0108 & -0.0033 \\
\hline Minimum & -0.2844 & -0.2030 \\
\hline Maximum & 0.3270 & 0.1930 \\
\hline Standard Deviation & 0.0590 & 0.0352 \\
\hline Skewness & 0.690 & 0.299 \\
\hline Kurtosis & 5.97 & 6.27 \\
\hline
\end{tabular}

Thomson Reuters Datastream is also the source of the independent variables. We consider both lagged endogenous and exogenous selected among the Asian world stock indices that close right before the opening of the U.S. market in order to account for possible market sentiment on latest economic news or response to progress in major world affairs (see, e.g., Shen et al., 2012). Specifically, variables are selected by looking at their correlation with the VXF OTCR. Results suggest to keep lags 0 and 1 of the DRs of the following four indices: Nikkei 225 (N225), Hang Seng (HSI), ASX 200 (ASX200) and SENSEX (BSESN). To allow for a possible autoregressive dependence we also keep the first two lags of the dependent variable, even if the (linear) autocorrelation function was not significant. Values for non-lagged indices are available from 8.30 to 5 hours prior to the opening of the CBOE. Therefore, we try to exploit as much as possible the information available to traders in their "nowcasting" activity, assuming that they need a minimum time lag in order to estimate models and set up their investment strategies. We do not consider data from European markets.

Data also exhibit significant cross-correlations at higher order for BSESN and N225, but we do not take into account this in accordance with the parsimony principle. We also exclude macroeconomics, bonds or commodity because their informative content is often questioned in the literature (see, e.g., Psaradellis and Sermpinis, 2016).

In summary, we work with four independent variables, namely the DRs of the Nikkei 225, Hang Seng, ASX 200 and SENSEX. Standard $t, \mathrm{JB}, \mathrm{ADF}, \mathrm{PP}, \mathrm{KPSS}$ tests show that each of these variables has zero mean and is normally distributed and mean-stationary (at the 95\% confidence level). On the overall, if we count both lagged and coincident variables, we perform an initial specification step by considering, besides the intercept, ten regressors (those reported in Table 2) for both the neural network and the logistic models and fifteen regressors (those reported in Table 3) for the HAR_X model.

\section{Results}

\subsection{Model specification and estimation}

Let us consider the logistic regression (5) with the above 10 regressors. This model, which we call $L 10$, is estimated by maximum likelihood on the sub-sample of data containing the first $T_{2}=2086$ observations. As we may note (see Table 2), not all the variables turn out to be significant. Thus, after a backward stepwise selection process, only the current BSESN is retained (together with the intercept), since it is the only significant variable. We call this parsimonious model L1. It is worth noticing that BSESN contains the most recent information, since India is the market that closes last among the four we considered. $L 10$ shows a better goodness-of-fit than $L 1$ but it has a worse BIC, and thus the less parametrized model turns out to be our choice.

In order to account for a more general form of non-linearity, we also estimate a feed-forward single hidden layer neural network. Analogously to what done for the logistic specification, we consider two sets of inputs, namely all the 10 variables appearing in Table 2 and the current BSESN only. Models are labelled as $N 10_{J}$ and $N 1_{J}$, respectively, where $J$ represents the number of neurons in the hidden layer and is chosen according to the 
procedure outlined below. For both the model with one and with ten regressors we will find the best value of $J$ based on the cross entropy (4).

Table 2. Parameters' estimations (benchmark models on VXF)

\begin{tabular}{c|c|c}
\hline \hline & $L 10$ & $L 1$ \\
\hline Intercept & $-0.2570 * *$ & $-0.250^{* * *}$ \\
$V X F$ OTC $R_{t-1}$ & -0.0110 & \\
$V F X$ OTC $R_{t-2}$ & -0.0060 & \\
$A S X 200 D R_{t}$ & -0.0882 & \\
$A S X 200 D R_{t-1}$ & -0.0773 & \\
$B S E S N D R_{t}$ & $-0.1053 * *$ & $-0.0942^{* *}$ \\
$B S E S N D R_{t-1}$ & 0.0462 & \\
$H S I D R_{t}$ & 0.0385 & \\
$H S I D R_{t-1}$ & 0.0297 & \\
$N 225 D R_{t}$ & 0.0306 & \\
$N 225 D R_{t-1}$ & -0.0064 & \\
\hline$B I C$ & $2.9008 \times 10^{3}$ & $2.8635 \times 10^{3}$ \\
Pseudo $R^{2}$ & 0.0112 & 0.0068 \\
\hline \hline
\end{tabular}

The neural network is trained with the scaled conjugate gradient algorithm on the training set containing the first $T_{1}=1718$ observations, and we early stop the training algorithm based on the network performance achieved on the validation set containing $T_{2}-T_{1}=368$ observations. The maximum number of iterations of the conjugate gradient method is capped at 1000 .

For both the $N 1_{J}$ and the $N 10_{J}$ architectures, the number $J$ of neurons in the hidden layer is chosen as follows: first of all, we generate 10000 randomly selected sets of weights, which we use as initial weights to train the neural network. Then, we consider the following expected performance indices:

$$
E P\left(N \cdot{ }_{J}\right)=\frac{1}{10000} \sum_{i=1}^{10000} M D A_{i}\left(N \cdot{ }_{J}\right)
$$

where $M D A_{i}$ is the mean directional accuracy (computed according to (10), with $O T C R_{t}$ for $t=1,2, \ldots, T_{2}$ ) that we obtain when we train the neural network starting from the $i$-th set of weights.

We let $J$ vary from 2 to 20 and we find the value that maximizes the $E P$ in (18). For both the $N 1_{J}$ and the $N 10_{J}$ specifications, the maximum $E P(N \cdot J)$ value is obtained with $J=16$ neurons, which we thus identify as the best complexity for the two models. The expected performance of the network with only $B S E S N_{t}$ as input, 16 hidden nodes and 2 output neurons is $E P\left(N 1_{16}\right)=59.7 \%$, whereas the expected performance of the network with all the ten regressors is $E P\left(N 10_{16}\right)=58.4 \%$. Moreover, once 16 neurons in the hidden layer are chosen, we also compute the maximum of the expected performance over the set of 10000 sets of initial weights:

$$
E P_{\max }\left(N \cdot{ }_{16}\right)=\max _{i=1,2, \ldots, 10000} M D A_{i}\left(N \cdot{ }_{16}\right)
$$

Both the $N 1_{16}$ and the $N 10_{16}$ networks reach the same maximum $M D A$, equal to $61.4 \%$, while the maximum $M D A$ achieved by the logistic specifications $L 1$ and $L 10$ is lower, namely $59.2 \%$ and $60.2 \%$, respectively. 
Moreover, the maximum $M D A$ achieved by $H A R, H A R_{-} X_{6}$ and $H A R_{-} X_{15}$ is $49.4 \%, 53.0 \%$ and $52.8 \%$, respectively.

The gap of accuracy between the neural networks and the logistic models is essentially due to the greater ability of the networks to grasp upwards movements (those less frequent in the sample but more profitable, as their average OTCR is $2.64 \%$ vs. an average OTCR of $-2.50 \%$ for negative returns). In particular, if we focus on upward returns, the maximum values of $M D A$ reached by $N 1$ and $N 10$ are $37.8 \%$ and $41.0 \%$, respectively, while the logistic specification performs only 38 true positives on 910 positive observations. By contrast, the neural network is superior to the HAR class in forecasting the downward movements of the VXF.

All over considered, the neural networks yield levels of prediction accuracy that are considerably higher than those achieved by other models, which indicates the existence of a marked non-linearity in the relationship between futures OCTRs and the exogenous variables.

If we agree on the fact that predictive performances constitute an effective measure of informative contents, then markets are capable to pack the information about past events into current information. That is, the dependence on the most recent indicator (the BSESN index) subsumes the information contained in all the other (less recent) variables. This conclusion holds for both the logistic specification and the neural network model, so it is robust across different types of non-linearity in the relationship linking variables. Furthermore, our finding that all the relevant information about the current events is contained in the most recent past is consistent with several empirical studies (see, e.g., Ahoniemi, 2006 and Degiannakis, 2008) showing the low predictive accuracy of time series models with long memory. For all of these reasons, only parsimonious specifications (with one input variable) of the neural network and logistic models are considered hereafter.

The estimation of the HAR and HAR_X models, which is shown in Table 3, provides a slightly different picture. The introduction of the exogenous variables still brings a clear improvement of the BIC but now, unlike what we experienced for the logistic model, the coincident BSESN is not the only informative variable, as also the lagged endogenous and indices from other markets than India are statistically significant.

Table 3. Parame te rs' estimations (benchmark models on VIX)

\begin{tabular}{c|c|c:c}
\hline \hline & $H A R \_X_{15}$ & $H A R \_X_{6}$ & $H A R$ \\
\hline Intercept & $0.4524^{* *}$ & $0.3377^{* *}$ & $0.282^{* *}$ \\
$V I X D R_{t-1}$ & $0.5898^{* *}$ & $0.7122^{* *}$ & $0.7913^{* *}$ \\
$M A(V I X D R)_{t-1}^{5}$ & $0.3340^{* *}$ & $0.2368^{* *}$ & $0.1820^{* *}$ \\
$M A(V I X D R)_{t-1}^{22}$ & $0.0564^{* *}$ & $0.0365^{*}$ & 0.0140 \\
$B S E S N D R_{t}$ & $-0.3055^{* *}$ & $-0.4020^{* *}$ & \\
$M A(B S E S N D R)_{t}^{5}$ & 0.0137 & -0.1151 & \\
$M A(B S E S N D R)_{t}^{22}$ & 0.0993 & -0.1586 & \\
$A S X 200 D R_{t}$ & $-0.2168^{* *}$ & & \\
$M A(A S X 200 D R)_{t}^{5}$ & $-0.3076^{*}$ & & \\
$M A(A S X 200 D R)_{t}^{22}$ & 0.4116 & & \\
$H S I D R_{t}$ & -0.0602 & & \\
$M A(H S I D R)_{t}^{5}$ & 0.0112 & & \\
$M A(H S I D R)_{t}^{22}$ & $-0.4622^{*}$ & & \\
$N 225 D R_{t}$ & -0.0387 & & \\
$M A(N 225 D R)_{t}^{5}$ & $-0.2180^{*}$ & & \\
$M A(N 225 D R)_{t}^{22}$ & -0.2569 & & \\
\hline$B I C$ & $8.5321 \times 10^{3}$ & $8.5771 \times 10^{3}$ & $8.7977 \times 10^{3}$ \\
$R^{2}$ & 0.9683 & 0.9665 & 0.9624 \\
\hline \hline & & & \\
\hline \hline
\end{tabular}


Moreover, the BIC of $H A R \_X_{15}$ is slightly better than the BIC of $H A R \_X_{6}$. Nevertheless, in order to perform a broader comparisons with the $L 1$ and the $N 1_{16}$ specifications, we consider not only the best performing $H A R \_X_{15}$, but also $H A R \_X_{6}$ with BSESN as the only exogenous regressor, and the $H A R$ as a benchmark.

\subsection{Assessing forecasting performance}

We then shift the focus on out of sample performance by considering the set of data from day $T_{2}+1$ to day $T_{3}$. We find that the out-of sample exact classification rates of models $L 1, N 1_{16}, H A R \_X_{15}, H A R \_X_{6}$ and HAR are $60.2 \%, 65.8 \%, 52.4 \%, 52.4 \%$ and $42.1 \%$ respectively.

At the first glance, the performances of the models that include exogenous variables are in line with the exact classification rates obtained in previous works (i.e. 61.9\% in Ahoniemi, 2006; 55.4\% in Konstantinidi and Skiadopoulos, 2011; 70\% Degiannakis et al., 2018). However, it must be stressed out that a straight comparison is not possible without considering the frequency of observed negative OTCRs in the test sample.

This point becomes particularly important when working with VXFs, since negative returns are more frequent than positive returns As a hypothetical example, let us think to the case where the frequency of negative returns in the test sample is $70 \%$. Then, a model that reaches an exact classification rate of $70 \%$ does not perform better than the Naïve model always forecasting negative outcomes.

Accordingly, we should measure the performance of the models in term of relative performance with respect to the observed frequency of negative OTCRs in the test set, which we find to be equal to $59.8 \%$. Noting that the observed frequency of negative OTCRs coincides with the $M D A$ of the Naïve model, we obtain a measure of relative performance $(R P)$ by subtracting the $M D A$ of the Naïve model from the $M D A$ of each of the competing specifications. In particular, the one input neural network achieves a relative performance $R P_{N 1}=$ $6.0 \%$ (i.e. $65.8 \%-59.8 \%$ ), the logistic specification reaches a relative performance $R P_{L 1}=0.4 \%$ (i.e. $60.2 \%$ $-59.8 \%$ ), while $H A R \_X_{15}, H A R \_X_{6}$ and $H A R$ perform worse than the Naïve specification.

One could argue that forecasting performances (relative to the Naïve model) depend how data are allocated among estimation, validation and test sets. In order to determine if our results are robust to sample partition, we make a Monte-Carlo cross validation robustness check for the models with positive relative performance. We consider 1000 random permutations of the sequence of days $1,2, \ldots, T_{3}$ at which data were observed. For each permuted sequence we continue to form the training set with the data at places $1,2, \ldots, T_{1}$, the validation set with the data at places $T_{1}+1, T_{1}+2, \ldots, T_{2}$, and the test set with the data at places $T_{2}+1, T_{2}+2, \ldots, T_{3}$. Then, we re-estimate both the $L 1$ and the $N 1_{16}$ models by using the data at places $1,2, \ldots, T_{2}$, (again, to select the neural network, we consider 10000 different random initial weights and we choose the neural network that achieves the best performance on the validation set). Finally, for each of the 1000 Monte Carlo sequences, we compute the $M D A$ performances of the models by using the data at places $T_{2}+1, T_{2}+2, \ldots, T_{3}$ as follows:

$$
\begin{gathered}
R P_{N 1}=M D A_{i}\left(N 1_{16}\right)-M D A(\text { Naïve }) \\
R P_{L 1}=M D A(L 1)-M D A(\text { Naïve })
\end{gathered}
$$

The distribution of the relative performance (over the 1000 random permutations) is reported in Figure 2. As we may observe, $R P_{N 1}$ is rarely negative, reaches a maximum value of $8.7 \%$, and its median is equal to $3.4 \%$. Moreover, the relative performance (6.0\%) which we experienced using the true (baseline) sequence of OTCR values corresponds to the $92.7^{\text {th }}$ percentile of the distribution of $R P_{N 1}$. 
The above evidence suggests that $N 1_{16}$ performs considerably better than the Naïve model (whose relative performance is null), and that such a conclusion is robust to sample allocation.

Figure 2. Out of sample relative performance, 1000 random pe rmutations of all the $T_{3}$ data. $R P_{N 1}(\mathrm{a})$; $R P_{L 1}(\mathbf{b})$.

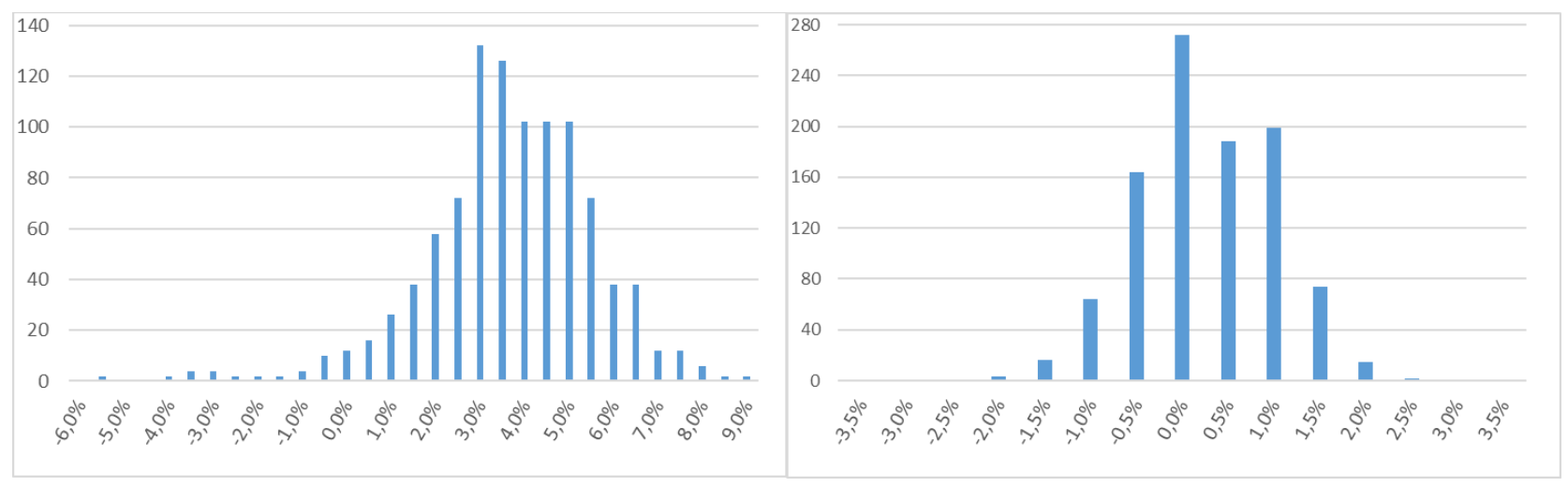

Finally, let us perform a model comparison by using both the PT and SPA tests described in Section 3.2. For the sake of brevity, we only consider the $L 1, N 1_{16}$ and Naïve specifications, since, as already observed, the $H A R \_X_{15}, H A R \_X_{6}$ and $H A R$ specifications perform worse than the Naïve specification.

The Hansen test is done considering, in turn, each of the $L 1, N 1_{16}$ and Naïve models as the benchmark (so as to check if eachmodel achieves a better prediction accuracy than the other five models). Moreover, to compute the test statistic $T^{S P A}$ (see (15)) we take the opposite of the $M D A$ as the loss function, and the p-values associated to $T^{S P A}$ are obtained based on 1000 bootstrap resamples. A small p-value indicates that the predictive accuracy of the model chosen as benchmark is inferior to the predictive accuracy of at least one of the alternative specifications.

Results are reported in Table 4. The PT test higlights that the market timing ability is different across models. In fact, it is possible to reject the null hypothesis of independence between true and forecasted direction of change only for model $N 1_{16}(p<1 \%$,), whereas for model $L 1$ the probability of making a type-I error is close to $10 \%$.

Table 4. Model performance and superior predictive ability

\begin{tabular}{ccc}
\hline \hline & $\begin{array}{c}\text { PT } \\
(\mathrm{p} \text {-value })\end{array}$ & $\begin{array}{c}\text { SPA } \\
(\mathrm{p} \text {-value })\end{array}$ \\
\hline Nä̈ve & $/ /$ & 0.007 \\
$L 1$ & 0.080 & 0.005 \\
$N 1_{16}$ & $1.35 \mathrm{E}-09$ & 1 \\
\hline \hline
\end{tabular}

Moreover, in accordance with our previous findings, the Hansen test confirms that $N 1_{16}$ is the only model providing systematic and sizeable improvements in forecast accuracy with respect to the other models. Then, if we agree that the predictive performance is an effective specification test (i.e. a measure of the informative value of the input variables and of the validity of the functional form of the models), the following conclusions hold. First, the most recent information (the current BSESN) encompasses the contribution of all the less recent variables, which is, actually, a form of weak efficiency that involves markets of different countries; second, the functional relation that links the VXF OTCR to the current BSESN is complex and non-linear. 


\subsection{Trading simulation}

Nevertheless, accuracy does not necessarily imply profitability. Thus, we also checked economic profits by simulating a simple trading strategy that amounts to either doing nothing or opening a long/short position when the market opens and liquidating it when the market closes.

In particular, when predicting with the $N 1_{16}$ and the $L 1$ models, trading is done as follows: at day $t$, before the opening of the CBOE, we forecast the probability that the VXF OTCR will be positive for that day, which, for the sake of brevity, we denote with $p_{t}^{+}$. Moreover, let THR denote a given threshold (filter). If $p_{t}^{+} \geq 0.5+$ $T H R$, then at day $t$ we take a long position on the $\operatorname{VXF}\left(O_{t}=1\right)$ when the market opens and we liquidate it when the market closes. If $p_{t}^{+} \leq 0.5-T H R$, then at day $t$ we take a short position on the $\operatorname{VXF}\left(O_{t}=-1\right)$ when the market opens and we liquidate it when the market closes. Finally, if $0.5-T H R<p_{t}^{+}<0.5+T H R$, then at day $t$ we stay flat $\left(O_{t}=0\right)$. The trading strategy applied to the $H A R \_X_{15}, H A R \_X_{6}$ and $H A R$ outputs is analogous to that described above, with the only exception that we take into account the magnitude of the forecasted DR on VIX. Specifically, THR is now the smallest magnitude of the forecasted DR on VIX that is required in order to open a long/short position on VXF. That is, if the magnitude of forecasted VIX DR does not exceed $T H R$, then at day $t$ we stay flat $\left(O_{t}=0\right)$.

Note that the threshold allows us to optimize the trading strategy and, consequently, it is determined based on the first $T_{2}$ observations, once the final models have been selected/estimated. In particular, following a common practice, we attempt to avoid "false signals" by simply filtering out the weakest signals. Precisely, as done by Ahoniemi's (2006), we consider six different threshold levels for each model (see Table 5) with the goal to find which filter yields the highest level of profitability on the first $T_{2}$ observations. As far as profitability is concerned, we measure it by means of the cumulative directional value (16) (where we replace $T_{2}$ and $T_{3}$ with 1 and $T_{2}$, respectively). Bid-ask spread are neglected and, following a common approach (see, e.g., Psaradellis and Sermpinis, 2016), commissions are set to 50 cents each contract.

The results obtained are reported in Table 5. As we may observe, the neural network performs significantly better than the logistic model with every filter and it is also more profitable than the HAR, HAR_ $X_{15}, H A R \_X_{6}$ models provided that $T H R \leq 2.5 \%$. The augmented HAR models are more profitable than the simple HAR model with any filter, which further confirms the importance of leveraging the time zone difference when collecting exogenous information. The neural network is the only model that achieve the best trading performance with $T H R=0$, i.e. that can correctly discriminate even the weakest signals from the BSESN.

Table 5. Profitability for different probability thresholds in the time period from day 1 to day $T_{2}$ (in parenthesis the fraction of trading days in the considered time period)

\begin{tabular}{|c|c|c|c|c|c|c|c|}
\hline & $\overline{N N 1_{16}}$ & $\overline{L L 1}$ & & $\overline{\bar{H}}$ & $\overline{4 R}$ & 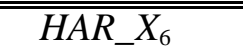 & "HAR_X $X_{15}$ \\
\hline$T H R=0$ & $663.5 \%(100 \%)$ & $308.0 \% \quad(100 \%)$ & $T H R=0$ & $-33.9 \%$ & $(100 \%)$ & $433.2 \% \quad(100 \%)$ & $420.6 \% \quad(100 \%)$ \\
\hline$T H R=0.5 \%$ & $622.0 \%(95.4 \%)$ & $312.6 \%(96.0 \%)$ & $T H R=0.25 \%$ & $-78.3 \%$ & $(87.5 \%)$ & $372.8 \%(93.7 . \%)$ & $429.8 \%(94.2 \%)$ \\
\hline$T H R=1 \%$ & $641.1 \%(93.5 \%)$ & $323.3 \%(94.4 \%)$ & $T H R=0.5 \%$ & $-7.5 \%$ & $(75.6 \%)$ & $374.9 \%(87.0 \%)$ & $448.5 \%(87.7 \%)$ \\
\hline$T H R=2.5 \%$ & $639.9 \%(87.5 \%)$ & $384.8 \%(89.2 \%)$ & $T H R=1 \%$ & $65.0 \%$ & $(50.7 \%)$ & $422.8 \%(72.4 \%)$ & $462.9 \%(75.2 \%)$ \\
\hline$T H R=5 \%$ & $537.6 \%(77.6 \%)$ & $458.6 \%(70.5 \%)$ & $T H R=1.5 \%$ & $99.2 \%$ & $(32.2 \%)$ & $433.6 \%(59.7 \%)$ & $412.2 \%(65.1 \%)$ \\
\hline$T H R=10 \%$ & $362.4 \%(46.6 \%)$ & $82.7 \%(10.0 \%)$ & $T H R=2 \%$ & $-25.9 \%$ & $(18.4 \%)$ & $349.9 \%(48.9 \%)$ & $384.1 \%(54.0 \%)$ \\
\hline
\end{tabular}

Furthermore, the HAR_X models achieve performances that are quite similar to those of the logistic specification. They perform a large amount of true positives, which is crucial for profitability, as the highest 
VXF OTCRs are usually experienced in correspondence of upward movements. On the contrary, the logistic is the specification that performs best in predicting true negatives, with an exact classification rate greater than $92 \%$ for any $T H R \leq 5 \%$.

Once the filter (THR) that yields the optimal trading strategy is selected (for each specifications) we measure the out of sample profitability on the data observed at days $T_{2}+1, T_{2}+2, \ldots, T_{3}$. The goal is to check whether there are significant differences also in the economic performances of the best strategies that can be constructed based on the predictions of the neural network, logistic, Naïve, HAR and HAR_X models. This is accomplished by means of the SPA test, in which the opposite of $D V$ (see relation 17) is used as the loss function, and the p-values of the statistic $T^{S P A}$ are computed based on 1000 bootstrap resamples.

The results obtained are reported in Table 6 (the maximum drawdown in the 5-th column is, actually, the $C D V$ (16) computed by considering only losses reported in consecutive trading days). As we may observe, all the models except for HAR generate relevant profits. However, the Naïve model exhibits an extremely large maximum drawdown, which makes it impossible to match the performance reported in Table 5 by an investor who does not have substantial additional capital to compensate losses. It is interesting to observe that the neural network outperforms all the competitors in terms of profitability, and it also yields the smallest number of false signals.

If we consider risk (measured by the standard deviation of returns and by the maximum drawdown), the strategy built on the forecasts of the logistic regression yields the best performance, but the result depends on the applied filter: in particular, the smallest standard deviation and the smallest maximum drawdown are achieved if the percentage of effective trading days is $80 \%$ (see the second column of Table 6). Nevertheless, the strategy based on neural network forecasts has the highest Sharpe ratio, i.e. it yields the best trade-off between expected profit and risk. Finally, as far as the HAR class is concerned, we may observe that the performances of $H A R \_X_{6}$ and $H A R \_X_{15}$ are very similar, and that they are much more profitable than $H A R$.

The SPA test confirms, on an inferential base, that the $N 1_{16}$ neural network provides systematic improvements in economic performance over all the other models. We conclude that, by taking into account information with a minimum time zone difference and by using a very flexible non-linear specification, we can achieve significantly higher profits with respect to the logistic, the Naïve, the HAR and the HAR_X models.

Table 6. Trading strategies' performance and Supe rior Pre dictive Ability in the time period from day $T_{2}+1$ to day $T_{3}$ (in parenthesis the fraction of trading days in the considered time period)

\begin{tabular}{|c|c|c|c|c|c|}
\hline models & $\begin{array}{c}\text { profitability } \\
(C D V)\end{array}$ & $\begin{array}{c}\text { \# false signals } \\
(1-M D A) \\
\end{array}$ & $\begin{array}{c}\text { Returns' } \\
\text { standard dev. }\end{array}$ & $\begin{array}{l}\text { Maximum } \\
\text { drawdown }\end{array}$ & $\begin{array}{l}\text { SPA test } \\
\text { (p-value) }\end{array}$ \\
\hline Nä̈ve & $\begin{array}{l}226.9 \% \\
(100 \%)\end{array}$ & $40.1 \%$ & $4.3 \%$ & $53.4 \%$ & 0.012 \\
\hline$L 1$ & $\begin{array}{c}466.8 \% \\
(80 \%)\end{array}$ & $34.2 \%$ & $3.8 \%$ & $15.1 \%$ & 0.028 \\
\hline$N 1_{16}$ & $\begin{array}{l}647.8 \% \\
(100 \%)\end{array}$ & $34.2 \%$ & $4.2 \%$ & $24.7 \%$ & 1 \\
\hline$H A R$ & $\begin{array}{c}17.4 \% \\
(49.4 \%)\end{array}$ & $59.0 \%$ & $4.6 \%$ & $49.0 \%$ & 0 \\
\hline$H A R \_X_{6}$ & $\begin{array}{l}326.3 \% \\
(62.7 \%)\end{array}$ & $47.6 \%$ & $4.3 \%$ & $16.4 \%$ & 0.003 \\
\hline$H A R \_X_{15}$ & $\begin{array}{l}317.5 \% \\
(78.3 \%)\end{array}$ & $47.6 \%$ & $4.3 \%$ & $19.3 \%$ & 0.005 \\
\hline
\end{tabular}




\subsection{Trading simulation based on daily returns $(D R s)$}

In order to provide additional information more comparable with the existing literature, we assess the profitability of the previous models when the dependent variable is chosen to be the VXF DR, rather than the VXF OTCR.

Following the same procedure as in Section 4.1, first of all we select the more informative exogenous variables in the logistic model as well as the best neural network architecture. Results indicate that two coincident variables, namely the BSESN DR and the ASX200 DR, and two lagged variables, namely the VXF DR and the BSESN DR, are statistically significant in explaining the DRs dynamics. We denote the logistic model with those regressors as $L 4$. We use the same information set to train the network, finding that the best architecture is now achieved by employing 10 nodes in the single hidden layer. We indicate this optimal architecture as $N 4_{10}$. For the sake of comparison, we also calculate the performance of the HAR, HAR_X $H A R \_X_{15}$ models considering the same values of THR as in Table 5.

The results obtained (with the optimally chosen filter) are reported in Table 7. Again, the neural network turns out to be the best performing model, yielding profits that are at least 1.7 times higher than those provided by any of the rival specifications. Moreover, the models that directly predict the VXF are more profitable if they are used with OTCRs rather the DRs (compare with Table 6). The gap is even more evident if we consider the sharp ratio. On the contrary, models that forecast the VIX direction (the HAR class), yield higher profits when the trading strategies are based on DRs rather than OTCRs. However, if we focus on risk, the standard deviation of profits is on the overall larger than that experienced in the case of OTCRs, which reflects the higher level of uncertainty that affects DRs.

Finally, the SPA testconfirms, on an inferential base, that the neural network provides systematic improvement over all the other models considered.

Table 7. Trading strategies' performance and Superior Pre dictive Ability in the time period from day $T_{2}+1$ to day $T_{3}$ (in parenthesis the fraction of trading days in the considered time period).

\begin{tabular}{|c|c|c|c|c|c|}
\hline $\begin{array}{l}\text { models } \\
\text { on DRs }\end{array}$ & $\begin{array}{c}\text { profitability } \\
(C D V)\end{array}$ & $\begin{array}{c}\text { \# false signals } \\
(1-M D A)\end{array}$ & $\begin{array}{c}\text { Returns' } \\
\text { standard dev. }\end{array}$ & $\begin{array}{l}\text { Maximum } \\
\text { drawdown }\end{array}$ & $\begin{array}{l}\text { SPA test } \\
\text { (p-value) }\end{array}$ \\
\hline Nä̈ve & $\begin{array}{l}47.4 \% \\
(100 \%)\end{array}$ & $41.2 \%$ & $4.7 \%$ & $52.2 \%$ & 0 \\
\hline$L 4$ & $\begin{array}{l}324.9 \% \\
(85.9 \%)\end{array}$ & $40.4 \%$ & $4.7 \%$ & $21.2 \%$ & 0 \\
\hline$N 4_{10}$ & $\begin{array}{l}632.9 \% \\
(100 \%)\end{array}$ & $36.7 \%$ & $4.5 \%$ & $21.1 \%$ & 1 \\
\hline$H A R$ & $\begin{array}{c}78.6 \% \\
(49.4 \%)\end{array}$ & $60.1 \%$ & $4.9 \%$ & $47.7 \%$ & 0 \\
\hline$H A R \_X_{6}$ & $\begin{array}{l}336.6 \% \\
(62.7 \%)\end{array}$ & $49.0 \%$ & $4.7 \%$ & $18.4 \%$ & 0.016 \\
\hline$H A R \_X_{15}$ & $\begin{array}{l}349.5 \% \\
(78.3 \%) \\
\end{array}$ & $49.4 \%$ & $4.6 \%$ & $27.2 \%$ & 0.020 \\
\hline
\end{tabular}

Moreover, BSSEN, despite remaining the only variable that is significant at both the coincident and lagged levels, is no longer capable to explain DRs by its own (as the coincident ASX200 DR and the lagged VXF DR 
are found statistically significant). This is due to the fact that the dynamics of DRs is more complex than that of OTCRs, since it is also influenced by nonsynchronous trading effects and bid-ask bounces (see Anderson et al., 2012).

It is also interesting to note that, if the trading strategy is based on VXF OTCRs rather then on VXF DRs, the performance of the Naïve model deteriorates significantly. In fact, the high profitability of the Naïve specification that we obtained by considering OTCRs (226.9\%, see Table 6) is essentially due to the fact that the volatility of financial markets (and hence also the VIX index) is normally greater in the morning and smaller at night (see, e.g., Daigler, 1998 and Garcia et al., 2018), so that selling the VXF in the morning and buying it at night turns out to be a profitable trading strategy. Instead, when taking into account VXF DRs, such an intraday effect is not exploited any longer, because trading is done only when the market closes and the regularity of the volatility trend is somehow broken by the random information flow that arrives overnight (see Anderson et al., 2012).

Finally, even if the neural network achieves almost the same performances when considering DRs in place of OTCRs, the use of DRs implies that traders do not have a minimum time lag to forecast the VXF, and set up their investment strategies accordingly. In fact they should place the order before the market closes, when the closure price of the VXF (or of the VIX if they forecast with the HAR class of models), is still unknown. Consequently, when taking into account DRs, profit assessment might not correctly mirror the performance of the trading strategy that is followed in practice. This does not occur if OTCRs are considered, because traders have all the time (overnight) to forecast the VXF (or the VIX) and to place the order.

\section{Conclusions}

We have investigated several relevant aspects related to the predictability of the VIX future (VXF). The use of open to close returns (OTCRs) " $\ldots$ offer the advantage of taking into account only the "genuine" autocorrelation that arises from partial price adjustment and time-varying risk premia ..." (Anderson et $a l$., 2012). Moreover, the focus on VXF is close to the perspective of the investors who recognize that implied volatility is tradable only as a futures contract.

The present paper contributes to the existing literature in several respects. First, we show that the dynamics of the VXF does not closely reflect that of the VIX index. This is in line with previous works, but our analysis does also highlight that the "VIX-VXF Puzzle" is more serious when measured on OTCRs than on DRs. In particular, the correlation between the intraday returns of VIX and VXF is only 0.767 if calculated it on the whole sample (2454 observations), and it is 0.771 if we take the average of the correlations computed on monthly sub-samples. On the top of that, the correlation distribution is quite erratic, so that an investor who wants to trade the VXF based on some, even very accurate, prediction of VIX intraday returns might not be able to earn significant profits.

Second, we establish that the neural network and logistic models whose only input variable is the most recent exogenous one are superior to the unconstrained models with ten (lagged and coincident) regressors. Precisely, the specification that includes only the BSESN index yields a lower value of BIC and a higher expected performance. That is, prices on the CBOE reflect the last publicly available subsumed by the Indian index, which is, actually, a form of weak efficiency that involves markets of different countries.

As well, one-day lagged endogenous variables are less informative than the price dynamics in a market closing right before the US market, even if we fit data with a very flexible approximator such as a neural network. By contrast, we get evidence that lagged variables increase the network's complexity and cause overfitting problems. Thus, we can conclude that, in order to reduce overfitting, limiting the number of input nodes in these "black-box" models is more effective than pruning the hidden layer units. Overall, our findings reinforce the scepticism, widespread in the literature, about the usefulness of a pure long memory time series approach to VXF (or VIX) forecasting. 
Third, we compare the proposed neural network model with a logistic regression, a HAR and a HAR_X models, and a Naïve model that always forecasts negative outcomes. Results indicate that the mean directional accuracy achieved by the neural network specification is significantly higher than that achieved by all the other models. Moreover, as revealed by Monte-Carlo cross validation, the better performance of the neural network is robust to sample selection bias. Then, first, non-linearity matters. Furthermore, if non-linearity is combined with information available on a market closing right before the U.S. market, a considerable degree of predictability of VXF OTCR signs can be obtained. Specifically, we correctly forecast directional changes in the $65.8 \%$ of the trading days, which, in our opinion, represents a fairly good predictive performance confirming the existence of strong non-linearities and supporting the use of a neural network.

Fourth, in line with the most recent literature, we assess the profitability of alternative trading strategies that rely on predictions of VXF directional changes. We find that the use of filters to limit false signals (leaving out the weakest signals) enhances profitability for all the employed models except for the neural network which is capable to exploit all the information contained in the coincident BSESN index. Overall, the neural network performance was $647.8 \%$ in almost three years (553 trading days), which is significantly higher than the performance of both the Naïve, the logistic, the HAR and the HAR_X models.

Finally, in the present paper we do not use data from European markets, since we assume that traders need a minimum time lag to estimate models and set up investment strategies. Moreover, we only focus on the future with the nearest expiration date, i.e. we do not take into account the term structure of the VXF. However, it could be interesting to consider also some European market index and/or futures with longer maturities. This could be the subject of a future work. 


\section{References}

Ahn J. J., Kim D. H., Oh K. J. \& Kim, T. Y. (2012). "Applying option Greeks to directional forecasting of implied volatility in the options market: An intelligent approach". Expert Systems with Applications, 39, 9315-9322.

Ahoniemi K. (2006). "Modeling and forecasting implied volatility: An econometric analysis of the VIX index". Working paper, Helsinki School of Economics.

Anderson R. M., Eom K. S., Hahn S. B. \& Park, J. H. (2012). "Sources of stock return autocorrelation". Working paper, University of California at Berkeley, available at https://eml.berkeley.edu// anderson/Sources-042212.pdf.

Asensio I. O. (2013). “The VIX-VIX Futures Puzzle". Working paper, University of Victoria, available at https $/ /$ www.uvic.ca/socialsciences/economics/assets/docs/seminars/Asensio.pdf.

Blaskowitz O. \& Herwartz H. (2011). "On economic evaluation of directional forecasts". International Journal of Forecasting, 27, 1058-1065.

Busch T., Christensen B. J. \& Nielsen M. Ø. (2011). "The role of implied volatility in forecasting future realized volatility and jumps in foreign exchange, stock, and bond markets". Journal of Econometrics, 160, 48-57.

CBOE (2015). "Chicago Board Options Exchange. The CBOE Volatility Index - VIX". CBOE White Paper, available at: 'http:/www.cboe.com/micro/vix/vixwhite.pdf'.

Corsi A. (2009). "A simple approximate long-memory model of realized volatility". Journal of Financial Econometrics, 7, 174-196.

Costantini M., Cuaresma J. C. \& Hlouskova J. (2016). "Forecasting errors, directional accuracy and profitability of currency trading: The case of EUR/USD exchange rate". Journal of Forecasting, 35, 652-668.

Daigler, R. T. (1998). "Intraday futures volatility and theories of market behavior". The Journal of Futures Markets, 17, 45-74.

Degiannakis S. A. (2008). "Forecasting VIX”. Journal of Money, Investment and Banking, 4, 5-19.

Degiannakis S. A. \& Filis G. (2017). "Forecasting oil price realized volatility using information channels from other asset classes". Journal of International Money and Finance, 76, 28-49.

Degiannakis S. A., Filis G. \& Hassani H. (2018). "Forecasting global stock market implied volatility indices". Journal of Empirical Finance, 46, 111-129.

Diebold F. X. \& Mariano R. S. (1995). "Comparing Predictive Accuracy”. Journal of Business \& Economic Statistics, 13, 134-44.

Fernandes M., Medeiros M. C., \& Scharth M. (2014). "Modeling and predicting the CBOE market volatility index". Journal of Banking and Finance, 40, 1-10.

Garcia C., Martelli A., Rona L. \& Ta A. (2018). "Intraday volatility prediction”. Working paper, available at https $/ /$ www.researchgate.net/publication/325678573_Intraday_Volatility_Prediction.

Granger C. W. J. \& Pesaran M. H. (2000). "Economic and statistical measures of forecast accuracy”. Journal of Forecasting, 19, 537-560.

Hansen P. R. (2005). “A test for superior predictive ability”. Journal of Business and Economic Statistics, 23, 365-380. 
Jabłecki J., Kokoszczyński R., Sakowski P., Ślepaczuk R. \& Wójcik P. (2014). "Does historical VIX term structure contain valuable information for predicting VIX futures?” Dynamic Econometric Models, 14, 5-28.

Konstantinidi E., Skiadopoulos G. \& Tzagkaraki E. (2008). "Can the evolution of implied volatility be forecasted? Evidence from European and US implied volatility indices". Journal of Banking and Finance, 32, 2401-2411.

Konstantinidi E. \& Skiadopoulos G. (2011). "Are VIX futures prices predictable? An empirical investigation". International Journal of Forecasting, 27, 543-560.

Leitch G. \& Tanner J. E. (1995). "Professional economic forecasts: Are they worth their costs?". Journal of Forecasting, 14, 143-157.

Leland H. E. (1999). "Beyond mean-variance: performance measurement in a nonsymmetrical world". Financial Analysts Journal, 55, 27-35.

Luo X. \& Zhang J. E. (2012). “The term structure of VIX”. Journal of Futures Markets, 32, 1092-1123.

Muravyev D. \& Ni X. (2016). "Why do option returns change sign from day to night?". Working paper, available at 'https://papers.ssrn.com/sol3/papers.cfm?abstract_id=2820264'.

Psaradellis I. \& Sermpinis G. (2016). "Modelling and trading the U.S. implied volatility indices. Evidence from the VIX, VXN and VXD indices". International Journal of Forecasting, 32, 1268-1283.

Shen S., Jiang H. \& Zhang T. (2012). "Stock market forecasting using machine learning algorithms”. Working paper, Department of Electrical Engineering, Stanford University, Stanford, CA, 1-5.

Shu J. \& Zhang J. E. (2012). "Causality in the VIX futuress market”. The Journal of Futures Markets, 32, 2446.

Thenmozhi M. (2006). "Forecasting stock index returns using neural networks". Delhi Business Review, 7, $59-69$.

Thomson Reuters Datastream(2010). "Futures Continuous Series - Methodology and Definitions". Working paper, available at 'http://zeerovery.nl/blogfiles/Datastream_Product_Futures_Continuous_Series.pdf'

Timmerman A. \& Pesaran M. (1992). "A simple nonparametric test of predictive performance". Journal of Business and Economic Statistics, 10, 461-465.

Whaley R. E. (1993). “Derivatives on market volatility: Hedging tools long overdue”. Journal of Derivatives, $1,71-84$.

Whaley R. E. (2008). “Understanding the VIX”. Journal of Portfolio Management, 35, 98-10. 